\title{
Remote Biosurveillance System for Water Bodies
}

${\text { Prakash Kodali }{ }^{1,2^{*}} \text {, Sanil K Danial }}^{2,3}$, NS Dinesh ${ }^{3}$ and NV Chalapathi Rao ${ }^{3}$

${ }^{1}$ Electronics and Communication Engineering Department, National Institute of Technology, Warangal, Telangana state, India

${ }^{2}$ Department of Electronic Systems Engineering, Indian Institute of Science, Bangalore, India

${ }^{3}$ Electronics and Communication Engineering Department, Government Engineering College, Kerala, India

*Corresponding author: Prakash Kodali, Electronics and Communication Engineering Department, National Institute of Technology, Warangal, Telangana State, India, Tel: +91-7353766700; E-mail: kprakash@nitw.ac.in

Received Date: May 17, 2018; Accepted Date: May 31, 2018; Published Date: June 12, 2018

Copyright: (C) 2018 Kodali P, et al. This is an open-access article distributed under the terms of the Creative Commons Attribution License, which permits unrestricted use, distribution, and reproduction in any medium, provided the original author and source are credited.

\begin{abstract}
A biological weapon appears to be more threatening than any other type of weapon, due to the psychological impact it creates in a society. The easiness of manufacture, less controllability and the quantum of calamity, make this an attractive tool for many extremist groups. The water distribution systems either build by nature (such as river, lake etc) or humanly made ones (such as a metro water distribution system, canals etc) are susceptible to biological attack. Biological agents (BA) are effective in very low doses. Therefore, Biological Agent Detection Systems (BADS) need to exhibit high sensitivity. The complex and rapidly changing environmental background also requires these detection systems to exhibit a high degree of selectivity (i.e. Able to discriminate BA from other harmless biological and non-biological material present in the environment). In this paper we present a Remotely Accessible Bio Surveillance System (RABSS) that can sense presence of the BA such as Bacteria in remote water bodies. The system is capable of detecting the presence of bacteria in water bodies that contains a cell count greater than or equal to ten. The fully automated system includes, a Sample Handling System (SHS), Sensor module, Wireless communication link and an embedded system to control all the functionalities in real time.
\end{abstract}

Keywords: Biological agents (BA); Biological agent detection systems (BADS); Remotely accessible Biosurveillance system (RABSS); Sample handling system (SHS)

\section{Introduction}

Biological weapons are more threatening than other warfare. There has been a remarkable increase in the production and availability of biological weapons throughout the world. Less infrastructure required for massive production and the quantum of calamity with less dosage are causing serious threats both from inside and outside to countries all over the globe. The weapons are centered on the Biological Agents (BA) that causes diseases to the people [1]. The agents can be Bacterial agents, viral agents, Rickettsiae and Biological toxins. The growth of these agents in various medium under favorable condition is exponential [2]. And moreover the activation period of these weapons are more and this gives enough time margin for the people involved in the attack to escape from the affected area. BA can easily be dispersed through medium such as air, food and water. BA is effective in very low doses. Therefore, Biological Agent Detection Systems (BADS) need to exhibit high sensitivity (i.e., be able to detect very small amounts of biological agents) and high degree of selectivity (i.e., be able to discriminate biological agents from other harmless biological and nonbiological material present in the environment) [1]. Water Distribution System (WDS) is one of the major susceptible networks for various types of attacks in world. Wide range of WDS can be easily polluted by BA such as Bacteria agents. Indeed, a bacterial culture depends on many factors such as external temperature, $\mathrm{pH}$ value, hygrometric rate, duration, and potential pollution levels [3-5].

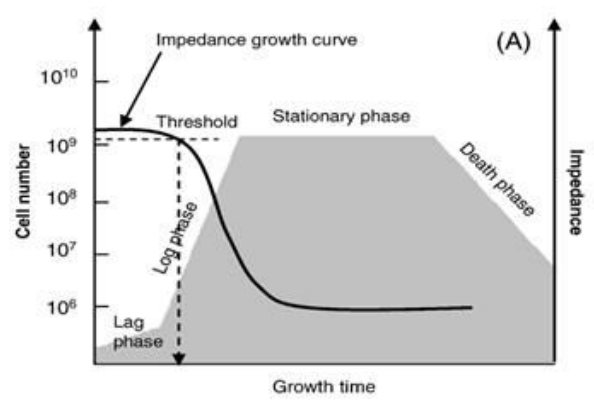

Figure 1: A generalized impedance growth curve.

\section{Experimental}

\section{Review of biological agent detection technologies}

Impedance technique : In this technique, changes in electrical impedance resulting from the bacterial growth is monitored. The impedance change is measured using a pair of electrodes merged in the cultured medium. Chemical changes happening in the medium due to bacteria metabolism, changes the conductivity of the medium. This acts as the basis for the impedance technique. The impedance systems usually measure the relative or absolute changes in conductance, capacitance, or impedance, at regular time intervals during the growth of bacteria at a given temperature. A typical impedance growth curve is illustrated in Figure 1. 
Amperiometric technique : Bacteria detection is done by directly measuring the current contribution of the bacteria immobilized on two adjacent $\mathrm{Au}$ electrode as shown in Figure 2. Consistent current measurement done typically in Pico amperes range helps in detecting even a single cell of bacterium in a controlled experiment.

The conduction is dominated by the interaction between $\mathrm{Au}$ electrodes and moisture remained on E. coli cells [6] and this can give wrong results in field equipment's.

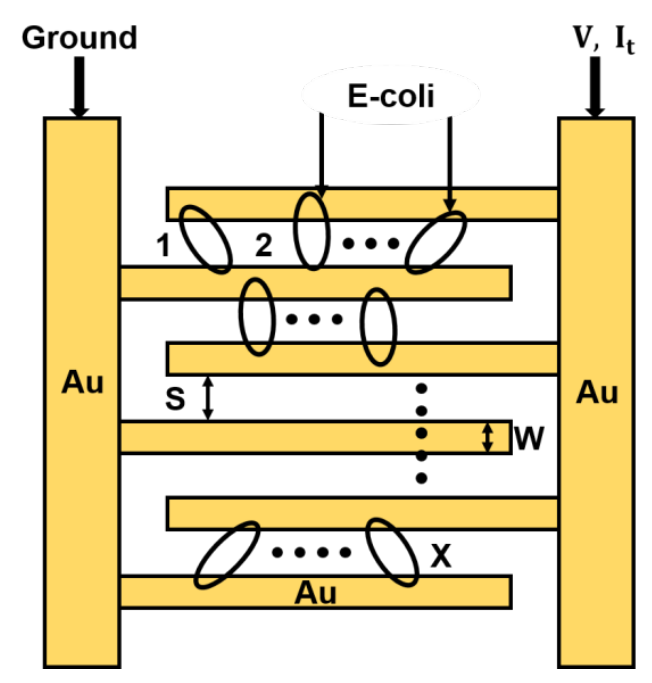

Figure 2 : Schematic of Au electrode arrays.

Nucleic acid based techniques: Nucleic Acid Based techniques Nucleic acid based techniques are used to detect the presence of DNA (Deoxyribo Nucleic Acid) or RNA (Ribo Nucleic Acid) of BA (nucleic acid amplification cannot directly detect the presence of the toxins themselves). The unique nucleic acid sequence, either DNA or RNA is used for the identification of agents.

Polymerase chain reaction (PCR) is the most commonly used nucleic acid based method for identification and detection of BA. It uses the enzyme DNA polymerase and short single stranded DNA primers to produce large quantities of identical copies of a specified sequence. The potential for detecting less than 10 copies of a specific gene within a complex sample makes PCR a popular molecular tool These techniques are generally not adaptable to field applications and require skilled personal for sample preparation [7].

Fluorescence technique: Fluorescence approaches involve excitation of molecular components of a material with light, usually in the ultra violet (UV) region of the spectrum. The excited component spontaneously reverts to an unexcited state followed by emission of light at different wavelengths. The emission spectrum is specific to the molecular component being irradiated and the excitation wavelength This phenomenon when applied in the detection of BA is termed as bio fluorescence. Fluorescent polymers added with bacteria samples enhances the level of fluorescence detection [8]. "A system has been developed at the Centre d'Etudes du Bouchet in France to detect toxic gas1 and contaminated surfaces" $[9,10]$. In the Saha equation [11] lower relative standard deviation (RSD) values are measured and obtained when the same kinds of emission line (atomic or ionic) are used.

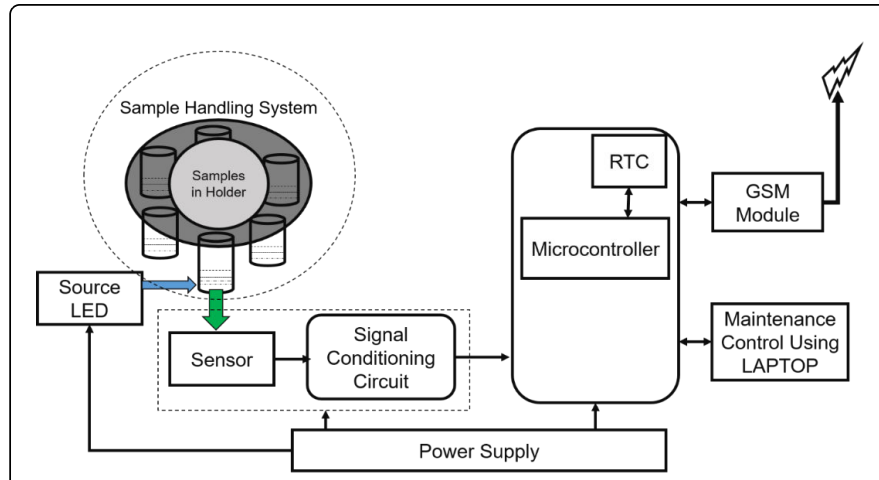

Figure 3 : Block schematic of remote surveillance system.

\section{Proposed Remote Surveillance System}

Remote Surveillance system can sense bacteria in water bodies. Lab equipment based on the discussed detection methodologies are available, the proposed system is a fully automated field equipment based on fluorescence approach. The system includes Sample Handling System (SHS), Sensor module, Master Controller and Communication module as shown in Figure 3. Operation of the system is fully automated right from sample collection and up to the transfer of the acquired information to a remote destination.

\section{Sample handling system}

A reservoir kept in the SHS holds the water samples from the water bodies under experimentation. SHS uses solenoid valves and separate reservoirs for medium and dye as shown in Figure 4. Sample handling involves the pre filtering of the water samples from the reservoir to filter out particles of larger dimension such as mud, leaves etc. The pre filtered water is flushed into an assay by operating a pump by keeping the valves V2, V3, V5, V6 closed and V1, V4 opened. The assay attached with membrane filter, filter out the bacteria present in the water. After filtering some volume (minimum $10 \mathrm{ml}$ ) of water, valve V1 is closed and valve V2 is opened to drain excess water droplets present in the assay. After switching on the pump and closing valve V2, valve V3 is opened. This is followed by opening valve V6 and then V5 to flush medium and dye respectively into the assay. The extracted particles are cultured in the medium. The medium after experimentation is flushed out of assay by operating the pump.

\section{Sensor module}

Remote bio surveillance system is a field equipment that can detect bacteria in water samples. Bio fluorescence approach is adopted for the detection of bacteria. The cultured Bacteria in SHS is allowed to grow for several hours. The sample holding assay is subjected to an excitation from a blue led whose emission spectrum is centered at 485 $\mathrm{nm}$. The output spectrum centered at $510 \mathrm{~nm}$ is fed to a photo transistor having relatively higher sensitivity at $510 \mathrm{~nm}$. Ac excitation is used in the sensor module to avoid dc errors. Sensor module is designed to work up to a relatively higher fluorescent dye concentration of 10 micro molar. As the bacteria grows in a medium that consists of both food and fluorescent dye, the concentration of dye in the medium increases with time. The increase in intensity of fluorescent light give more current variation and thereby more voltage 
Page 3 of 4

variation occurs across collector of the detector. This increase in voltage variation is quantified by taking the average value of the signal by using a precision rectifier as shown in Figure 5 .

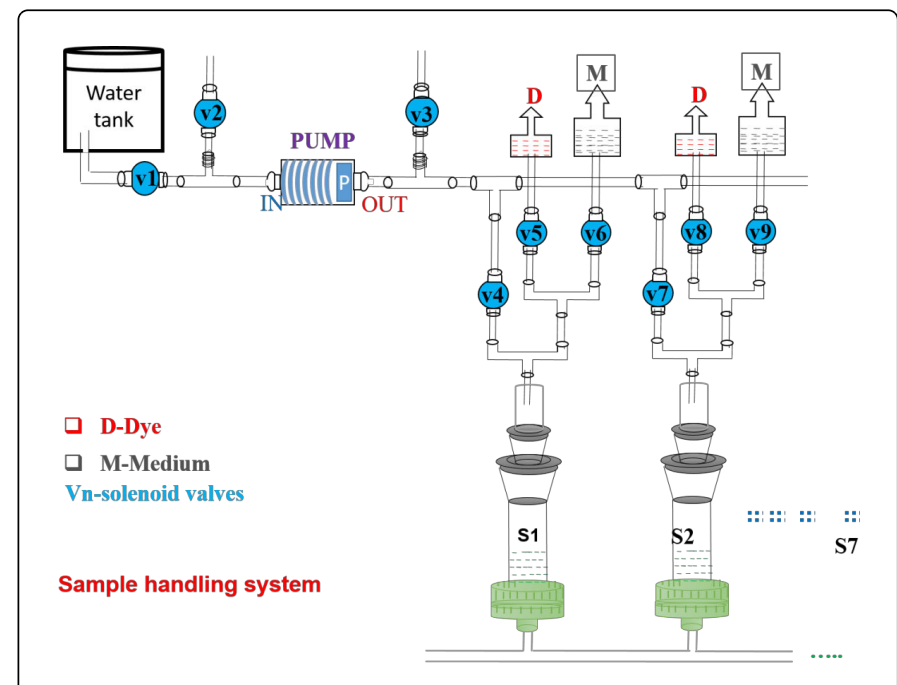

Figure 4 : Sample handling system.

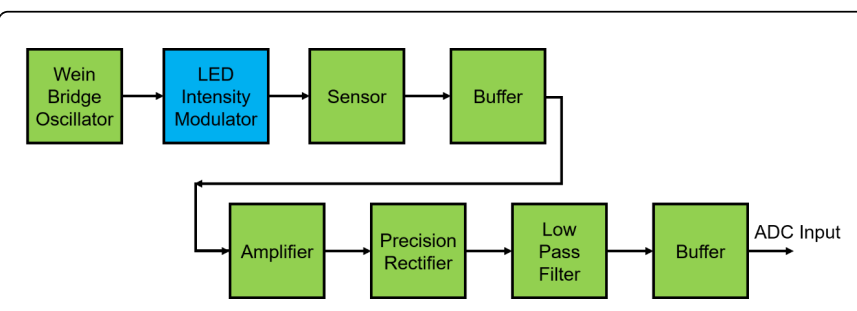

Figure 5: Block diagram of sensor module.

Relative measurement scheme is adopted for error compensation. Reading without source excitation (Re1), Reading with distilled water and source excited (Re2), and Reading with sample and source excited (Re3) are taken during the commencement and end of each and every sampling. Reading with blank (medium and dye) and excited source (Reb1 at the beginning and Reb2 after 12 hours) during very first experimentation is taken to find the fluorescence contribution due to dye hydrolysis and stored for future calculations. The difference between Re3 and Reb1 gives fluorescence contribution due to bacteria growth. The differences, (Re2-Re1) and (Re3-Reb1-Re1) gives readings free from the dye hydrolysis, dc errors if any and temperature variation respectively for the distilled water and the sample. Photo transistor used is subjected to gain variation with temperature and ageing and this is compensated by taking relative measurement. Initial relative reading, Rel1 (where Rel1 $=(\operatorname{Re} 3-\operatorname{Reb} 1-\operatorname{Re} 1) /(\operatorname{Re} 2-\operatorname{Re} 1))$ and a second set of relative reading, Rel 2 with values taken after a few hour is compared. Rel2 greater than Rel1 indicates the presence of bacteria in the background of other harmless biological materials.

\section{Master controller}

All the communication and the controlling of subsystems are happening through master controller. Master controller consists of microcontroller C8051F120, RTC (Real Time Controller) module and UART interface. Microcontroller used has an inbuilt ADC (Analog to
Digital Converter) of 12 bit. The analog data from the signal conditioning circuit is fed to the input pin of ADC port of the micro controller and it is converted to digital form. The lower nibble of 12 bit ADC output obtained, is found to be noisy and this is made smoother by averaging the digital output over 16 samples. The average digital value from various excitation schemes as explained in the previous section is stored for calculating the relative intensity variation. The excitation of sensor modules, sample handling operations and the controlling of communication modules are done by the master controller in real time with an interfaced RTC.

\section{Communication module}

Bio surveillance system designed is a fully automated field equipment that samples the water and sense the presence or absence of bacteria. The system is configured to do the experiments by taking samples at regular intervals in a day. Time management is done by the RTC connected to master controller. Acquired information is stored inside a flash memory and the information is transferred periodically to a remote destination via GSM. Any abnormalities in the level of bacteria in water bodies is remotely monitored. Information stored in flash memory is transferred to laptop through UART interface.

\section{Results and Discussion}

Experimentation with real bacteria samples of different initial cell count was conducted for 24 hours in lab condition. Initial cell count is varied in exponential powers of 10 starting from 101 to 107 . The samples are excited with $968 \mathrm{mV}$ peak to peak ac excitation. Results obtained are shown in Figure 6. Experiments are repeated in room temperature with similar excitation and results are compared with readings of Fluoroscan Ascent. Two sets of bacteria samples are used for the experiment. One set of sample i.e. :-sample 1 and sample 2 are kept at lab condition and the other set i.e. :-sample 3 and sample 4 are kept at room temperature. Initial, intermittent and final readings are taken.

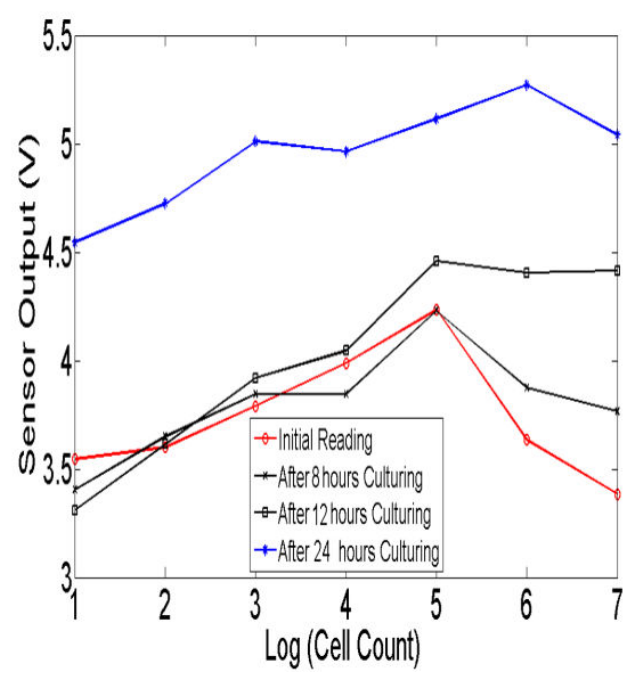

Figure 6 : Sensor output with Bacteria samples of different initial cell counts. 
Citation: Kodali P, Danial SK, Dinesh NS, Rao NVC (2018) Remote Biosurveillance System for Water Bodies. J Bioengineer \& Biomedical Sci 8:

Page 4 of 4

Comparison of readings after 12 hours is shown in Figures 7 (a) and 7 (b). Readings taken with sensor module closely matches with readings of Fluoroscan Ascent. Bacteria samples with an initial count of 102 was detected with the sensor module after twelve hours of culturing. An initial count of 10 cells maintained at lab conditions are reliably detected by the system. As shown in Figure 6, designed surveillance system deployed in remote field condition is able to detect even 10 cells of bacteria with 24 hours experimentation.

\section{Conclusion}

In this paper we present a remotely accessible Bio Surveillance system that can detect the presence of bacteria in remote water bodies. The system incorporates Sample Handling System, Sensor module, Master Controller and Communication module. Several bacteria detection techniques are available, but majority of the equipment build around these techniques are meant for laboratory applications. In our system bio fluorescence approach is adopted for the detection of bacteria. Designed system is able to reliably detect the presence of even ten numbers of bacteria in water at field condition with 24 hours of culturing. Operation of the system is fully automated right from sample collection and up to the transfer of the acquired information to a remote destination.

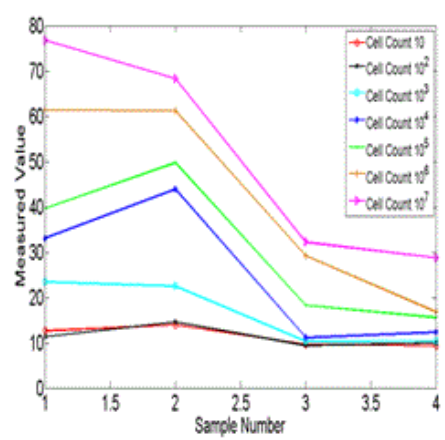

(a)

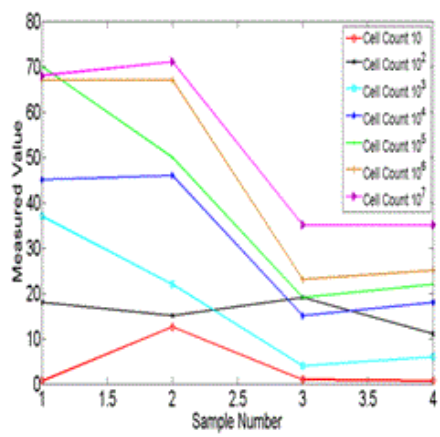

(b)

Figure 7 : Reading comparison after 12 hours of experimentation. (a) Relative fluorescence unit readings using fluoroscan ascent. (b) Relative measurement expressed in percentage using sensor module.

\section{References}

1. Stephane M, Nicolas L, Philippe A, Jacques A (2003) Detection of bacteria by time-resolved laser-induced breakdown spectroscopy. Appl Opt 42:30.

2. Liju Y, Rashid B (2008) Electrical/electrochemical impedance for rapid detection of foodborne pathogenic bacteria. Biotechnol Adv 26:135-150.

3. Alonso JM, Bejot J (1996) "Bacteries," in Encyclopoedia Universalis Encyclopædia Universalis S.A., Paris. pp: 719-735.

4. Stanier RV, Adelberg EA, Ingraham JL (1999) General Microbiology. Macmillan, London.

5. Neidhardt FC, Ingraham JL, Schaechter M (1994) Physiologie de la Cellule bacte'rienne : une approche moléculaire. Masson, Paris.

6. Yi-Chun L, Ya-Shuan C, Yi-Yang C, An-Chi S, Hsing-Yu H, et al. (2008) Bacteria detection utilizing electrical conductivity. Biosens Bioelectron 23: 1856-1861.

7. Douglas RC, Monica KB, Frank J L (2003) Detection of bacterial pathogens in environmental samples using DNA microarrays. J Microbiol Methods 53: 235-243.

8. Matthew DD, Juan Z, Timothy MS, Peter HS (2004) Detection of bacteria with carbohydrate-functionalized fluorescent polymers. J Am Chem Soc 126: 13343-13346.

9. Dudragne L, Adam Ph, Amouroux J (1998) Time-resolved laser-induced breakdown spectroscopy: Application for fluorine, chlorine, sulfur, and carbon in air. Appl Spectrosc 52: 1321-1327.

10. Dudragne L, Morel S, Adam P, Amouroux J (1999) Analysis of polluted surfaces by time-resolved laser-induced breakdown spectroscopy. Ann NY Acad Sci 891: 183-198.

11. Arago'n C, Aguilera JA, Peñ alba F (1999) Improvements in quantitative analysis of steel composition by laser-induced breakdown spectroscopy at atmospheric pressure using an infrared Nd:YAG laser. App Spectrosc 53: 1259-1267. 